\title{
Assessment of nutrition status in transplant patients using imaging, serologic markers and functional testing: Which best predicts early reversal of malnutrition?
}

\author{
$1^{\text {st }}$ Sam Atoa ${ }^{1}, 2^{\text {nd }}$ Richard S. Mangus ${ }^{2}$ \\ ${ }^{1}$ Indiana school of Medicine, Department of surgery, transplant section.
}

Background and Hypothesis: Previous studies have shown that patients with malnutrition have worse clinical outcomes which lead to longer hospital stays and increased costs. Little has been studied in regards forms of testing such as functional $5 \mathrm{M}$ walk tests and serologic testing in predicting nutritional status.

Experimental Design or Project Methods: Adults with end-stage liver, kidney, or pancreatic failure and with pre-transplant computed tomography (CT) imaging were selected from the transplant database. Each group was separated by organ and compared to each other by each nutritional measurement. Measures of nutrition status included a scaled scoring of core muscle mass, and visceral and subcutaneous fat stores, protein and albumin serum levels, BMI and sarcopenic index.

Results: It is clear that Liver patients started off much more malnourished than other organ groups as indicated by the low protein and albumin levels at day 0. Liver patients with severe sarcopenia continued to lose weight after 90 days despite increased albumin levels which suggests there is a rapid and significant loss of weight post-transplant due to diuresis which doesn't reverse until around day 60 .

Conclusion and Potential Impact: Serologic testing and imaging seemed to be good indicators of early reversal of malnutrition while functional testing did not. These finding will be analyzed against a control group to see which best predicts clinical outcomes such as length of post-operative hospital stays and fall risks. Being able to predict outcomes through nutritional status will help us to better prescreen high risk transplant candidates. 